\title{
Acoustic Relaxation of Liquid Poly(tetramethylene oxide) with Hydroxyl and Acyl Terminal Groups
}

\author{
Valeriy SHILOV, ${ }^{\dagger}$ Volodymyr SPERKACH, ${ }^{*}$ Yaroslav SPERKACH, ${ }^{*}$ \\ and Anatoliy STRYBULEVYCH,
}

\begin{abstract}
Institute of Macromolecular Chemistry, National Academy of Sciences of Ukraine, Kyiv 02160, Ukraine
*Physical Faculty, Kyiv National Taras Shevchenko University, MSP, 6 Gluskova Ave, Kyiv 03680, Ukraine
\end{abstract}

(Received April 16, 2001; Accepted June 25, 2002)

\begin{abstract}
Isotherms of sound absorption and velocity $v s$. frequency were measured for poly(tetramethylene oxide)-2000 ended by $\mathrm{CH}_{3}$ and $\mathrm{OH}$ groups. The measurements were performed at 5-2500 MHz and at different temperatures at $313-353 \mathrm{~K}$. The results are presented and analysed using different formats to examine the frequency dependence of the attenuation $\alpha(f) / f^{2}=f(f)$ and $\mu(f)=\alpha_{\text {exc }}(f) \lambda$ (where $\alpha(f)$ is sound attenuation at frequency $f$, $\alpha_{\mathrm{exc}}$ is excess sound attenuation at frequency $f$, and $\lambda$ is the sound wavelength) as well as frequency dependence of real $\left(M^{\prime}\right)$ and imaginary $\left(M^{\prime \prime}\right)$ parts of the mechanic modulus. Two dispersion regions are present on all sound absorption isotherms. Arrhenius curves of the relaxation time are fitted and analysed using Vogel-Tamann-Fulcher and Kohlrausch-WilliamsWatts functions. Relaxation time in the first dispersion region, as well as relaxation strength, are less affected by the terminal groups of PTMO than in the second dispersion region. Alternation of the different end-capping groups has greater influence on the relaxation strength values of the second dispersion region characteristic of the local segmental motions of the polymers.
\end{abstract}

KEY WORDS Poly(tetramethylene oxide)/ Ultrasonic Spectra / Relaxation Time /

The simplest aliphatic polyethers such as poly(methylene oxide) (PMO), poly(ethylene oxide) (PEO), poly(propylene oxide) (PPO), and poly(tetramethylene oxide) (PTMO) are well known polymers and widely used for industry and fundamental scientific interest. However, PPO, because of its noncrystallizability and availability in a wide molecular weight range, is acceptable as a model object for close examination of relaxation properties of flexible chain polymers. Its relaxation characteristics, basically those of the corresponding low molecular weight $\mathrm{OH}$-ended analogue, so-called poly(propylene glycol) (PPG), have been intensively studied using dielectric,${ }^{1-6}$ optical, ${ }^{7-10}$ nuclear magnetic resonance (NMR),${ }^{1-13}$ quasi-elastic neutron scattering, ${ }^{14,15}$ and ultrasonic, ${ }^{16-21}$ techniques, as well as various methods for probing viscoelastic properties. ${ }^{22-24}$

In contrast to PPO, PEO and PTMO are possible to crystallize and they can be closely investigated only in a temperature range above the melting point. Consequently, dynamics of such polyethers in their liquid/amorphous state is studied to a far lesser degree than that of PPG. There are a vast number of papers devoted to investigation of the relaxation features of OH-group end-capped low molecular weight PEO, poly(ethylene glycol) (PEG). The corresponding results are obtained using dielectric, ${ }^{25-27} \mathrm{NMR},{ }^{28}$ Brillouin scattering, ${ }^{29}$ and ultrasonic, ${ }^{30-32}$ techniques.

PPGs and PEGs are characterised for the most part by a presence of two room temperature dispersion regions, corresponding to the relaxation times of $10^{-8}$ and $10^{-10} \mathrm{~s}$. Those of PEG and PPG are rather close to each other at the same temperatures.

It follows from the one paper ${ }^{33}$ on relaxation behaviour of PTMO in a liquid state that room temperature quasielastic neutron spectra correspond to a relaxation time of $0.44 \times 10^{-10} \mathrm{~s}$. Some isolated experiments have been carried out to study relaxation characteristics of the amorphous regions of semi-crystalline PTMO using dielectrical, ${ }^{34,35}$ and mechanical ${ }^{36}$ relaxation techniques. It should be emphasised that PTMO is characterised by the smallest $T_{\mathrm{g}}, 178 \mathrm{~K}$. Correspondingly, its "cooperativity plot" (i.e., $\log \tau$ vs. $T_{\mathrm{g}} / T$ dependence) ${ }^{37}$ has the smallest steep slope of the corresponding polyethers.

The molecular motions of the polyethers of the molecular weight about thousand g per mol may be grouped as:

-Relaxation processes related to Rouse normal mode motions (RM) with the relaxation time, ${ }^{38} \tau_{\mathrm{RM}}$, at room temperature of the order of $10^{-8} \mathrm{~s}$.

-Local segmental motion (LSM) in the chain backbone ( $\alpha$-process) related to the dynamic glass transition, is always manifested in the relaxation spectra of

\footnotetext{
${ }^{\dagger}$ To whom correspondence should be addressed (Fax: +380-44-552-40-64, E-mail: shilov@ukrpack.net).

${ }^{\dagger}$ Present address: Ultrasonics Research Laboratory, Department of Physics and Astronomy, University of Manitoba, Winnipeg R3T 2N2, Canada, (E-mail: anatoliy@physics.umanitoba.ca).
} 
the polyethers. LSM, as opposed to RM, is independent of molecular weight and its room temperature relaxation time is $10^{-9}-10^{-10} \mathrm{~s}$.

Concerning PTMO, in addition to a considerable importance in fundamental science for investigation relaxation processes in polymers the corresponding OH-group capped PTMO (PTMG) is extensively used in production of polymers such as segmented polyurethanes due to their unique physical properties, ${ }^{39}$ ability to form water dispersions, ${ }^{40}$ and ionene-like structures ${ }^{41}$, ability to develop a single-ion transport mechanism, ${ }^{42,43}$ and ease of materials to meet the specific use requirements.

A distinctive feature of PTMG as well as other $\mathrm{OH}-$ group-ended polyethers is possibility to develop hydrogen bonds between the $\mathrm{OH}$ groups as well as $\mathrm{OH}$ groups and ether groups of repeated units. It was supposed, by Heinlich et al., ${ }^{44}$ that molecular chains of PPG are connected at the ends by hydrogen bonds of the end $\mathrm{OH}$ groups forming so-called transient entanglements should strongly influence the relaxation of the polyether. However, in the case of PPG such a supposition was not confirmed by later investigations. ${ }^{6}$ To consider the effects of hydrogen bonding on the relaxation of PTMG two PTMO-based liquids investigated. The first is OH-group end-capped PTMO, so-called poly(tetramethylene glycol), PTMG. The second, acylated PTMG named PTMA, is $-\mathrm{CH}_{3}$-group end-capped one. We normally think of acylated PTMO as a polymer not able to form, in contrast to PTMG, intermolecular $\mathrm{O}-\mathrm{H} \cdots \mathrm{O}$ hydrogen bonds and its relaxation features are not affected by intermolecular transient entanglements.

\section{EXPERIMENTAL}

PTMG, of nominal molecular weight 2000, was purchased from DuPont and used as received. To prepare PTMA, acetylation of PTMG was carried out by adding 1.5 times excess acetic anhydride $\left(\left(\mathrm{CH}_{3} \mathrm{CO}\right)_{2} \mathrm{O}\right)$ (Aldrich) $293 \mathrm{~K}$ and allowing the reaction to proceed with backflow condenser for $2 \mathrm{~h}$ at $413 \mathrm{~K}$. The product obtained was distilled under vacuum at $353 \mathrm{~K}$ and examined using IR spectroscopy.

Ultrasonic absorption measurements were performed at $313-353 \mathrm{~K}$ using pulse method at $5 \mathrm{MHz}-2.5 \mathrm{GHz}$ with an accuracy of $2 \cdots 5 \%$. The sound velocity was measured by a pulse-phase method with an accuracy of $0.1 \%$. Instruments and procedures are described elsewhere. ${ }^{45}$

Acoustic spectra obtained as involving two dispersion regions were resolved at each temperature, as first approximation, two Debye-like relaxation processes in accordance with the formula

$$
\alpha f^{-2}=\left(\frac{2 \pi^{2} c}{c_{0}^{2}}\right)\left[\frac{r_{1} \tau_{1}}{1+\left(\omega \tau_{1}\right)^{2}}+\frac{r_{2} \tau_{2}}{1+\left(\omega \tau_{2}\right)^{2}}\right]+B,
$$

where $\omega=2 \pi f ; c$ is frequently dependent sound velocity; $c_{0}$ low frequency sound velocity $\left(\omega \tau_{1}<<1\right) ; r_{i}$ and $\tau_{i}$ relaxation strength and relaxation time for the $i$ th single relaxation region $(i=1,2)$, respectively and $B$ high frequency limiting $\alpha f^{-2}$. To describe the frequency dependence of $\alpha f^{-2}$ the following empirical double relaxation equation was used

$$
\alpha f^{-2}=\frac{A_{1}}{1+\left(\omega \tau_{1}\right)^{2}}+\frac{A_{2}}{1+\left(\omega \tau_{2}\right)^{2}}+B .
$$

To determine $f_{i}=\left(2 \pi \tau_{i}\right)^{-1}$ and $A_{i}(i=1,2)$ and $B$ eq 2 was solved using the iteration method.

Ultrasonic spectra are expressed frequently in the, so-called $\alpha_{\text {exs }} \lambda$ format, using the function $\mu$ that is excess sound absorption per wavelength, ${ }^{46-48}$

$$
\mu=\alpha_{\mathrm{exc}} \lambda=\frac{\left(\alpha-B f^{2}\right) c}{f},
$$

where $\lambda=c / f$ is wavelength. In this case $\mu$ is evaluated as the sum of two relaxation processes by

$$
\mu=\frac{2 \mu_{1} f f_{1}^{-1}}{1+\left(f f_{1}^{-1}\right)^{2}}+\frac{2 \mu_{2} f f_{2}^{-1}}{1+\left(f f_{2}^{-1}\right)^{2}}
$$

$\mu_{1}$ and $\mu_{2}$ are maximum excess sound absorptions per wavelength at the respective relaxation frequencies $f_{1}$ and $f_{2}$. Comparing eqs 2 and 4 ,

$$
\mu_{1}=\frac{A_{1} f_{1} c}{2}
$$

and

$$
\mu_{2}=\frac{A_{2} f_{2} c}{2}
$$

Using eqs $1,2,4$, and 5 , one can take $r_{1}$ and $r_{2}$ as

$$
r_{1}=\frac{2 \mu_{1}}{\pi}=\frac{A_{1} f_{1} c}{\pi}
$$

and

$$
r_{2}=\frac{2 \mu_{2}}{\pi}=\frac{A_{2} f_{2} c}{\pi} .
$$

Frequency dependence of the real, $M^{\prime}$, and imaginary, $M^{\prime \prime}$, part of the longitudinal modulus, $M^{*}=$ $M^{\prime}+i M^{\prime \prime}$ are conventionally calculated from frequency dependence of ultrasonic absorption, $\alpha(f)$, and velocity, $c(f)$, as, ${ }^{49}$

$$
\begin{aligned}
M^{\prime} & =\rho c^{2} \\
M^{\prime \prime} & =\frac{2 \rho c^{3} \alpha}{\omega}
\end{aligned}
$$


Here $\rho$ is density.

Taking into account eq $3 M^{\prime \prime}$ is expressed as,

$$
\begin{aligned}
M^{\prime \prime} & =\frac{2 \rho c^{3}}{\omega}\left(\alpha_{\mathrm{exc}}+B f^{2}\right)=\frac{2 \rho c^{3}}{\omega} \alpha_{\mathrm{exc}}+\frac{\rho c^{3} f}{\pi} B \\
& =M_{\mathrm{exc}}^{\prime \prime}+\frac{\rho c^{3} f}{\pi} B
\end{aligned}
$$

$M_{\text {exc }}^{\prime \prime}$ represents the $\alpha_{\text {exc }}$-containing term and is more suitable for analysis in terms of width of relaxation time distribution than $M^{\prime \prime}$.

In most amorphous polymers, loss modulus data in the vicinity of the peak when plotted against $\log \omega$, can be fit well by the Fourier transform of a KohlrauschWilliams-Watts function (KWW).$^{50}$

$$
\Phi(t)=\exp \left[-\left(t / \tau_{i}\right)^{\beta}\right]
$$

where $0<\beta \leq 1$ width of distribution of relaxation time, being unity for a single relaxation time. $M^{\prime \prime}$ must be found as Fourier transformation of the $\Phi(t)$ function,

$$
M^{\prime \prime}=M_{\mathrm{r}} \int_{0}^{\infty} \cos (\omega t) \Phi(t) \mathrm{d} t
$$

to compare its profile with that calculated using acoustic spectroscopy data. Here $M_{\mathrm{r}}=M_{\infty}-M_{0}$ is the difference between high- and low-frequency limiting $M^{\prime}$.

\section{RESULTS}

Figures $1 \mathrm{a}$ and $1 \mathrm{~b}$ show ultrasonic absorption isotherms of PTMA and PTMG samples, respectively, as function of frequency in the temperature range studied. At $10-40 \mathrm{MHz}$ as well as $400-1500 \mathrm{MHz}$ all $\alpha / f^{2}$ vs. $f$ dependency curves clearly display dispersion characteristics and adopt constant $B$ at higher frequencies. Solid lines going through the measured values obey eq 4 and arrows in Figures 1a and 1b display positions of characteristic relaxation frequencies. In both samples, experimental $\alpha / f^{2}$ are represented satisfactorily by the double relaxation equation.

Ultrasonic spectra of our samples are expressed in terms of $\mu$ (eqs 3 and 4) and exhibited in Figures 2a and $2 b$. The arrows display two relaxation regions on curves of frequency dependence of excess absorption per wavelength. The absorption effect characteristic of the high frequency region exceed that presented in the low frequency region by too large a margin. All $\mu$ against frequency curves are represented, as first approximation, by the sum of two Debye relaxations (solid lines in Figure 2) using eq 4 . The relaxation frequency, $f_{1}$ and $f_{2}$, the values $A_{1}, A_{2}$, and $B$ parameters as well as $\mu_{1}$ and $\mu_{2}$ values are presented in Table I.

$f_{1}$ and $f_{2}$, of PTMA and PTMG samples shift to highfrequency with increasing temperature and $B$ decreases.

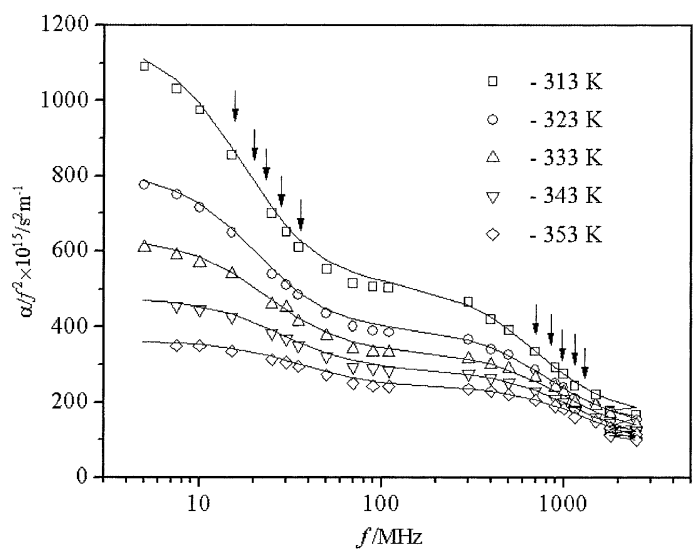

(a)

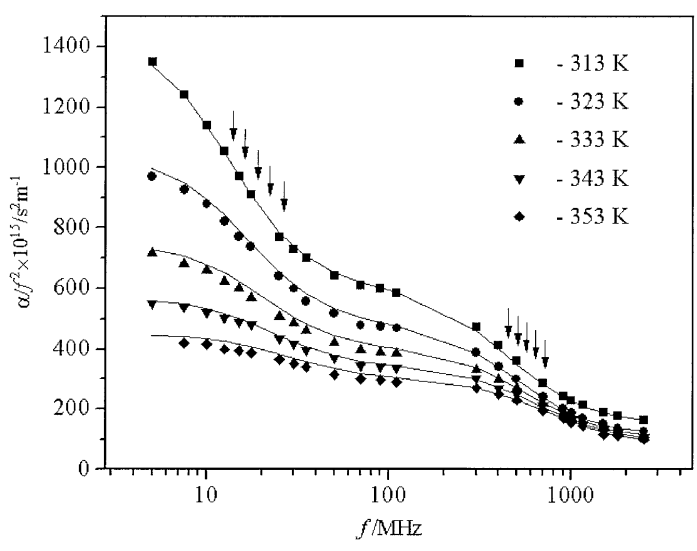

(b)

Figure 1. Frequency dependence of ultrasonic absorption of PTMA (a) and PTMG (b) at different temperatures. Arrows display positions of relaxation frequencies.

Replacement of $\mathrm{CH}_{3}$ end groups with $\mathrm{OH}$ leads to decreasing $f_{1}$ and $f_{2}$. In the case of $f_{2}$, the decrease is higher than for $f_{1} . \mu_{1}$ and $\mu_{2}$ decrease with increasing temperature. At 313 to $333 \mathrm{~K}, B$ of PTMG are lower than those of PTMA but after $343 \mathrm{~K}$ the reverse holds for $B$. In the temperature range considered $\mu_{2}$ far exceeded (by the factor from 20 to 50) those of $\mu_{1}$ and the higher is temperature, the greater is excess.

Sound velocity frequency dependence curves of PTMA and PTMG are presented in Figure 3. Marked sound velocity dispersion is characteristic of only the second relaxation frequency region.

The temperature dependency of the relaxation time of the first relaxation process, $\tau_{1}=1 /\left(2 \pi f_{1}\right)$, of PTMA and PTMG as well as that of the second relaxation are shown in Figure 4. $\tau_{1}$ vs. 1/T dependency curves of both PTMA and PTMG appear closely allied and show that, at near-room temperature conditions, $\tau_{1}$ are of the order of $10^{-8} \mathrm{~s}$. The temperature dependency of $\tau_{2}$ against $1 / T$ of PTMA and PTMG, contrastingly, is different and corresponds to the different room temperature $\tau_{2}$ of $1.78 \times 10^{-10}$ and $3.16 \times 10^{-10} \mathrm{~s}$, respectively.

A sizeable increase in $\tau_{2}$ is accounted for by the re- 


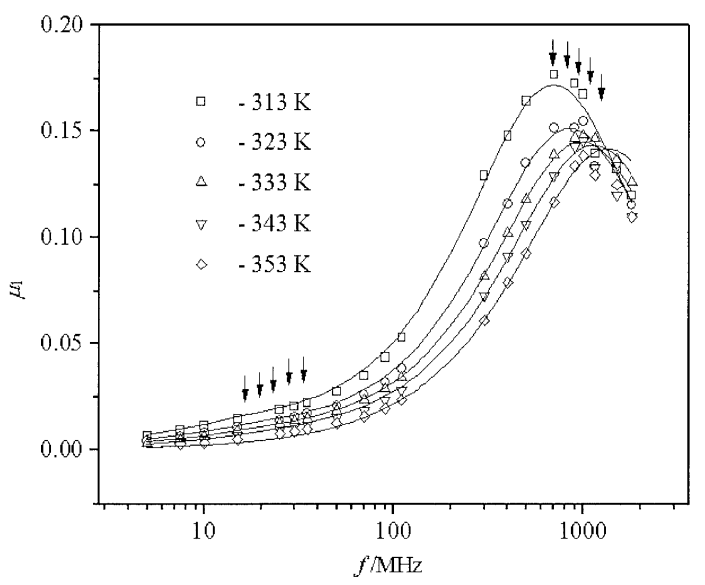

(a)

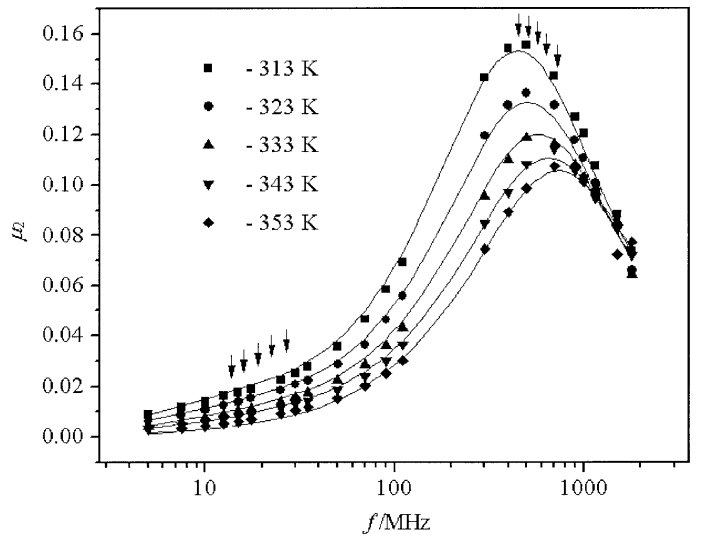

(b)

Figure 2. Frequency dependence of excessive absorption of PTMA (a) and PTMG (b) at different temperatures. Arrows display positions of relaxation frequencies.

Table I. Temperature dependence of relaxation of $\mathrm{CH}_{3}$ (PTMA) and OH-group (PTMG) end-capped low molecular weight PTMO

\begin{tabular}{cccccccc}
\hline$T, K$ & $A_{1}$ & $A_{2}$ & $B$ & $f_{1}$ & $f_{2}$ & $\mu_{1}$ & $\mu_{2}$ \\
\cline { 2 - 7 } & $\times 10^{15} \mathrm{Nps}^{2}$ & $\mathrm{~m}^{-1}$ & $\mathrm{MHz}$ & \multicolumn{1}{c}{$\times 10^{4}$} \\
\hline 313 & 850 & 470 & 150 & 14 & 450 & 85 & 1523 \\
323 & 560 & 370 & 110 & 17 & 510 & 66 & 1319 \\
333 & 350 & 300 & 100 & 20 & 580 & 47 & 1193 \\
343 & 230 & 250 & 90 & 23 & 655 & 35 & 1100 \\
353 & 150 & 220 & 80 & 28 & 740 & 27 & 1052 \\
\hline \multicolumn{7}{c}{ PTMA-2000 } \\
\hline 313 & 650 & 350 & 160 & 17 & 700 & 77 & 1711 \\
323 & 420 & 260 & 130 & 20 & 850 & 58 & 1507 \\
333 & 300 & 220 & 110 & 23.6 & 980 & 47 & 1444 \\
343 & 190 & 193 & 90 & 28.9 & 1140 & 36 & 1429 \\
353 & 120 & 166 & 75 & 35 & 1340 & 27 & 1413 \\
\hline
\end{tabular}

placement of $\mathrm{CH}_{3}$ end groups in the samples with $\mathrm{OH}$. In contrast $\tau_{1}$ increases a little in this case.

For polyethers the relaxation time $v s$. reciprocal temperature dependence obey, as a rule, the VogelTammann-Fulcher (VTF) equation. Although the temperature range, in which the acoustic relaxation time

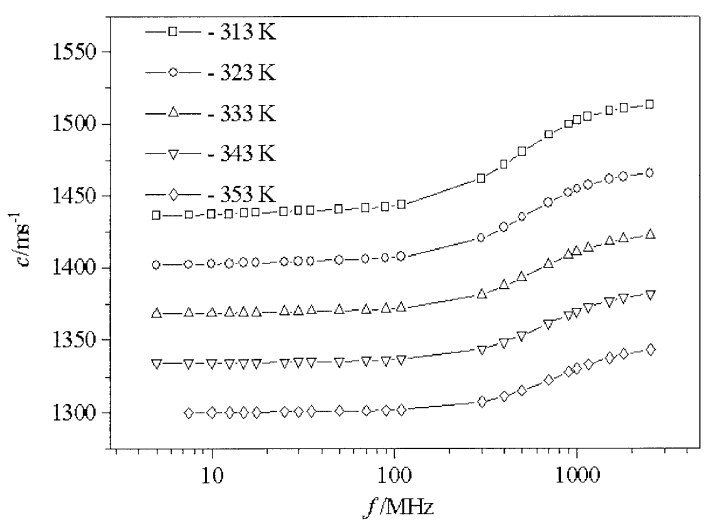

(a)

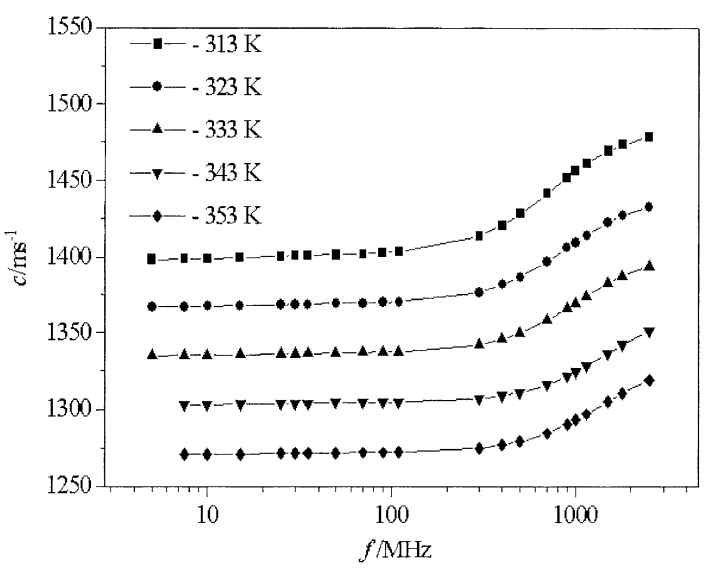

(b)

Figure 3. Frequency dependence of sound velocity of PTMA (a) and PTMG (b).

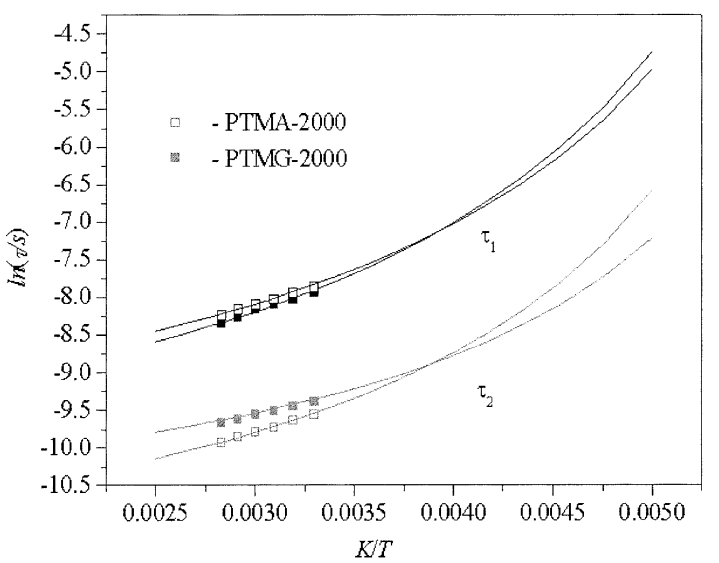

Figure 4. $1 / T$ dependence of $\tau_{1}$ and $\tau_{2}$ of PTMA and PTMG. The solid curves are the approximations by VFT-equation.

data are obtained, is not wide enough to confirm VTF behaviour we used such an equation to fit the temperature dependence of the $\tau_{1}$ and $\tau_{2}$.

$$
\tau_{i}=\tau_{0 i} \exp \left(\frac{B_{i}}{T-T_{0 i}}\right)
$$

Here $\tau_{0 i}, B_{i}$ and $T_{0 i}$ are adjustable parameters and $(i=$ $1,2)$.

We believe VTF approximation is useful for comparing acoustic relaxation data obtained with the relaxation 
Table II. Parameters for approximation of $1 / T$ dependency of $\tau_{1}$ and $\tau_{2}$ were fitted by use of the VFT equation. I* $^{*}$ and II* correspond to first and second relaxations, respectively

\begin{tabular}{ccccc}
\hline & \multicolumn{2}{c}{ PTMA-2000 } & \multicolumn{2}{c}{ PTMG-2000 } \\
\cline { 2 - 5 } & $\mathrm{I}^{*}$ & $\mathrm{II}^{*}$ & $\mathrm{I}^{*}$ & $\mathrm{II}^{*}$ \\
\hline $\log \left(\tau_{0}\right) / \mathrm{sec}$. & -8.95 & -10.33 & -8.69 & -9.7 \\
$B / K$ & 300 & 250 & 270 & 180 \\
$T_{0} / K$ & 140 & 145 & 140 & 145 \\
\hline
\end{tabular}

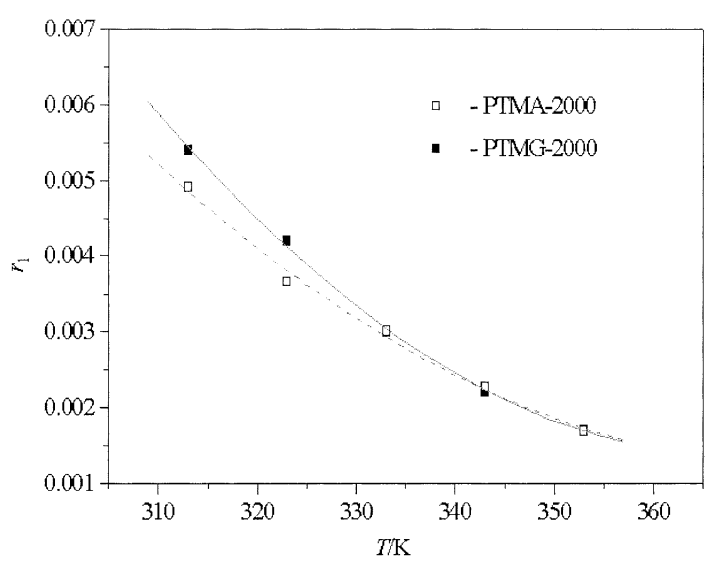

(a)

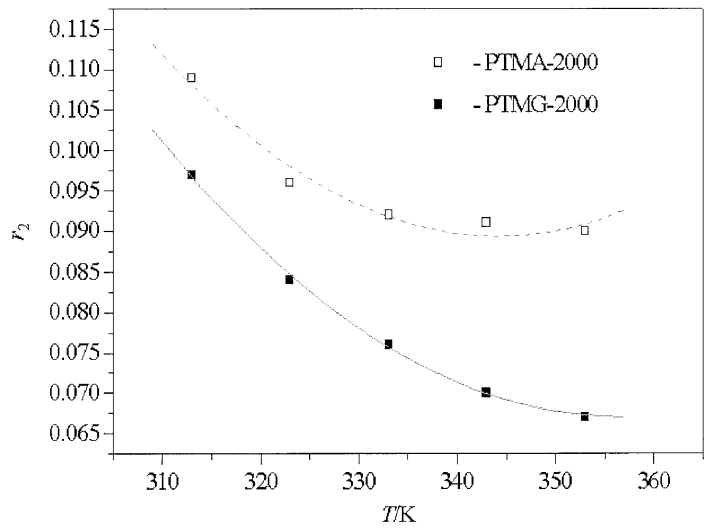

(b)

Figure 5. Temperature dependence of $r_{1}$ (a) and $r_{2}$ (b) of PTMA and PTMG.

data temperature dependence on PTMO obtained by other authors using different experimental techniques. VTF approximating curves are shown in Figure 4 by solid lines and $\tau_{0 i}, B_{i}$ and $T_{0 i}$ are listed in Table II. For the first relaxation the eq 14 parameters are altered but little after replacing $\mathrm{CH}_{3}$ end group with $\mathrm{OH}$. But $\tau_{02}$ and $B_{2}$ decreased markedly.

Figure 5a shows $r_{1}$ against temperature curves for PTMA and PTMG. $r_{2}$ vs. $T$ curves are drawn in Figure 5b. Relaxation strength of the first relaxation process, $r_{1}$, of PTMA and PTMG is a monotonically decreasing function of temperature. The corresponding relaxation strengths should follow,

$$
r_{1} \sim T^{-\delta}
$$

and exponents, $\delta$, obtained are of the order of 9 for Polym. J., Vol. 34, No. 8, 2002

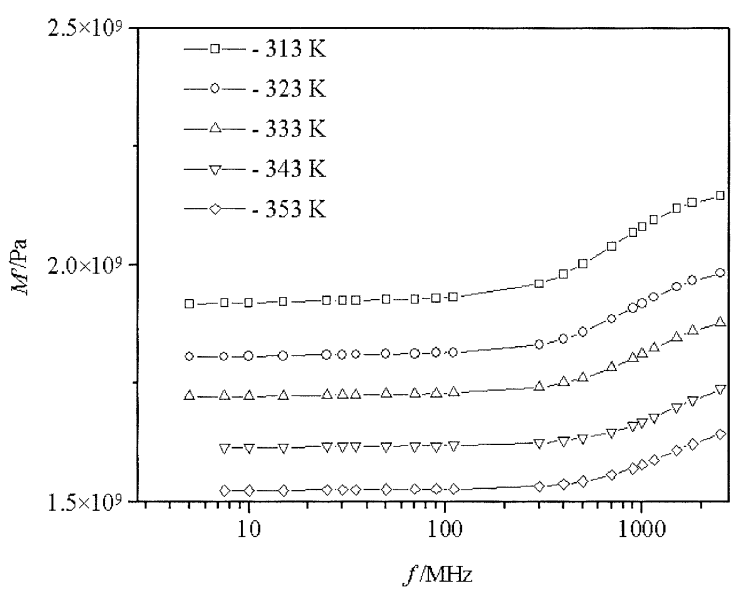

(a)

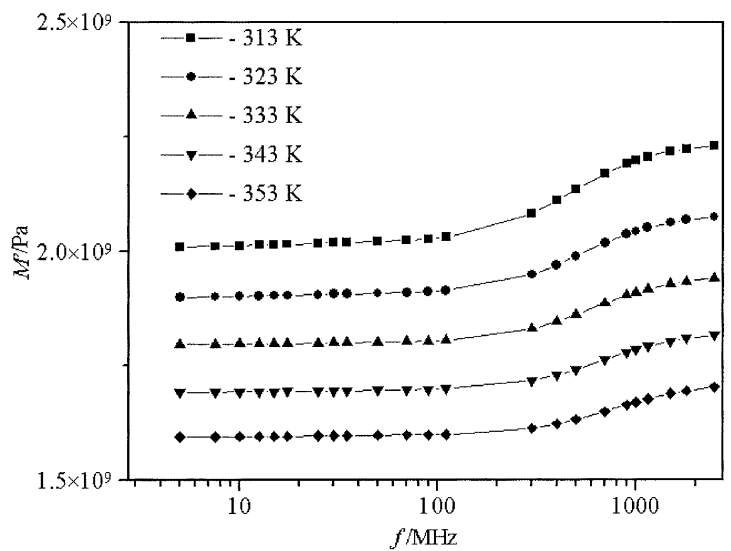

(b)

Figure 6. Frequency dependence of real modulus, $M^{\prime}$, of PTMA (a) and PTMG (b) at temperatures studied.

PTMA and PTMG.

The temperature behaviour of $r_{2}$ is different from that of $r_{1}$. First, for PTMA and PTMG, bi-logarithmic plots of the $r_{2}$ data, in distinction to those of $r_{1}$, are nonlinear in $T$. Second, the corresponding temperature dependencies of $r_{2}$ are far less steep in comparison with those of $r_{1}$. Thirdly, bi-logarithmic $r_{2} v s$. $T$ plots of PTMA are far less steep than for PTMG.

In general, the temperature behaviour of $\mu_{1}$ and $\mu_{2}$ should be similar that of $r_{1}$ and $r_{2}$. Comparing $r_{1}$ and $r_{2}$ as well as the $\mu_{1}$ and $\mu_{2}$ exhibit very strong distinctions. At all temperatures, $r_{2}\left(\mu_{2}\right)$ are far above the corresponding $r_{1}\left(\mu_{1}\right)$.

Frequency dependence of $M^{\prime}$ for PTMA and PTMG at the temperatures indicated are shown in Figure 6. The only jump, in the vicinity of $f_{2}$, occurs in a family of $M^{\prime} v s$. frequency isotherms. This is in line with the fact that in the case of PTMA as well as in PTMG the sole non-negligible velocity dispersion takes place.

Sets of $M^{\prime \prime}(f)$ for PTMA and PTMG are plotted in Figure 7. The solid lines represent $M^{\prime \prime}$ calculated according to the eq 10 from the approximating $\alpha / f^{2}$ curves of Figures 1 and 2. All of the corresponding 


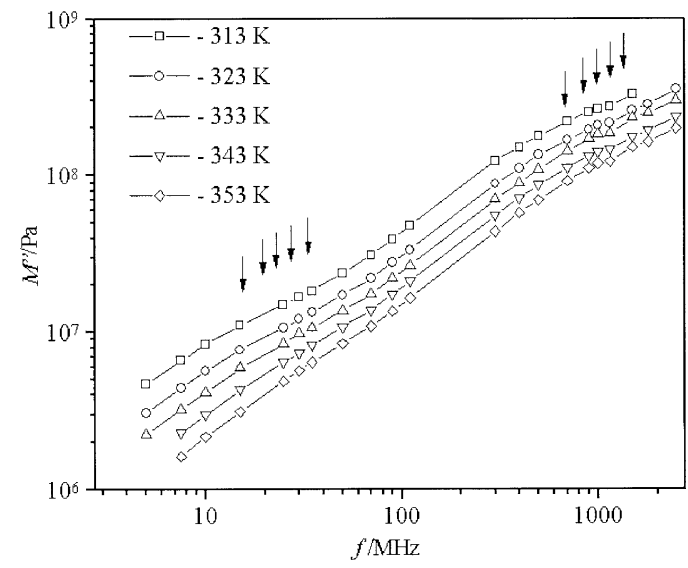

(a)

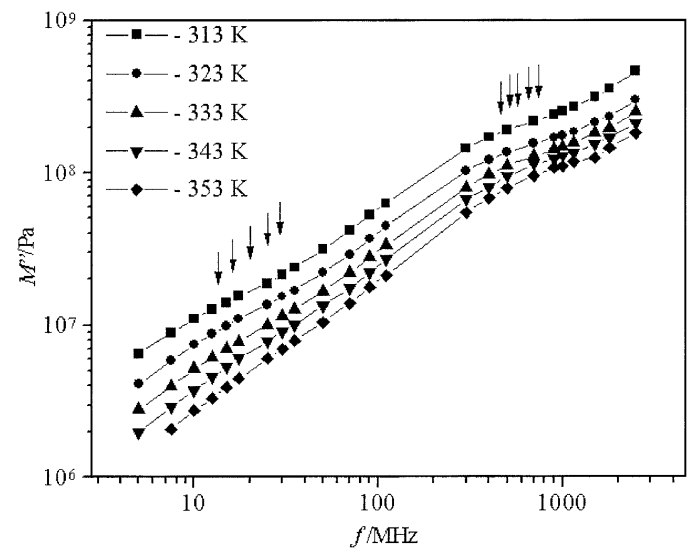

(b)

Figure 7. Frequency dependence of loss modulus, $M^{\prime \prime}$, of PTMA (a) and PTMG (b) at temperature range studied.

isotherms are characterised by two weakly pronounced maxima at frequency close enough to the corresponding $f_{1}$ and $f_{2}$ in Table $\mathrm{I}$.

To compare the frequency behaviour of $M^{\prime \prime}$ curves at different temperatures we reduced all to obtain a master plot to the $M^{\prime \prime}$ data at $313 \mathrm{~K}$, using the corresponding $f_{2}$ for determination of a horizontal displacement $\log \left(\frac{f_{2}^{\mathrm{T}}}{f_{2}^{313}}\right)\left(f_{2}^{313}\right.$ is $f_{2}$ at $T=313 \mathrm{~K}$ and $f_{2}^{\mathrm{T}}$ that at other temperatures used). The reduced $M^{\prime \prime}$ curves for PTMA and PTMG are presented in Figure 8. $M^{\prime \prime}$ data sets taken at various temperatures and reduced to the same temperature do not fall on a single composite curve for both polymers. Particularly striking is the considerable variation from one reduced curve to another at low frequency portions.

Relaxation characteristics can be investigated from plots of the imaginary modulus $M_{\mathrm{exc}}^{\prime \prime}$. Second $M_{\mathrm{exc}}^{\prime \prime}$ spectrum maxima of all the isotherms were normalized relative to each other to represent of them as $M_{\text {exc }}^{\prime \prime} / M_{\text {exc.max }} v s . \log \left(f / f_{2}\right)$. In this case $M_{\text {exc }}^{\prime \prime}$ is the frequency dependent $M^{\prime \prime}$ value and $M_{\text {exc.max }}^{\prime \prime}$ is the corresponding maximum of $M_{\mathrm{exc}}^{\prime \prime}$. The eq 13 was used to cal-

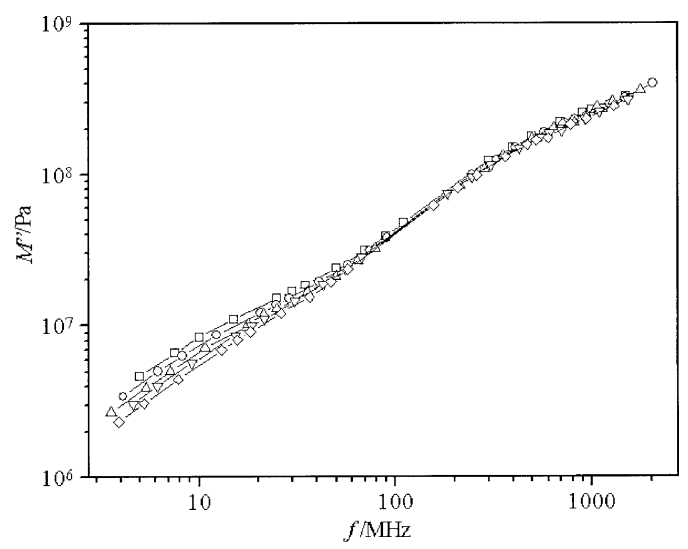

(a)

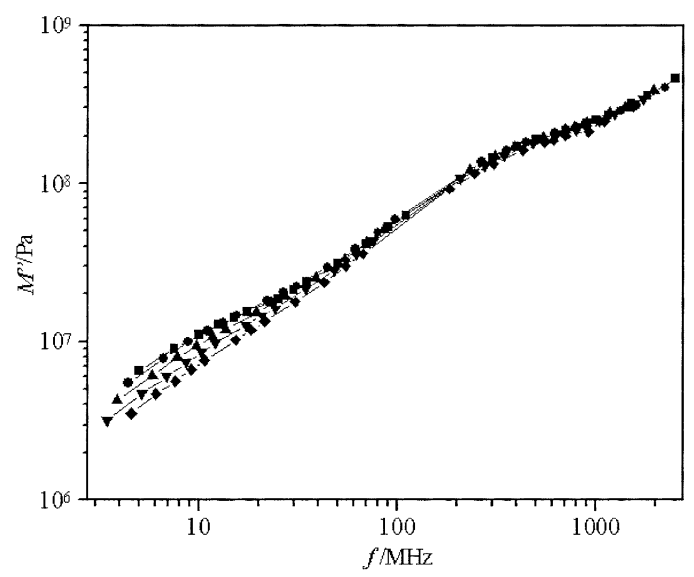

(b)

Figure 8. Loss modulus, $M^{\prime \prime}$, measurements on PTMA (a) and PTMG (b), reduced from different temperatures used to $313 \mathrm{~K}$ with shift factors calculated from a position of the high-frequency maximum.

culate the profile of the $\tau_{2}$-based maximum. The corresponding normalised $M_{\mathrm{exc}}^{\prime \prime}$ isotherm data for the second dispersion region fall on a single composite curve presented in Figures 9 (maximum the $M_{\text {exc }}^{\prime \prime} v s . f$ curves of PTMA (open symbols) and PTMG (solid symbols)). Model $M_{\mathrm{exc}}^{\prime \prime}$ spectra that were taken at different $\beta$, such as 0.7, 0.9, and 1.0, are presented in Figure 9. $\beta$ for PTMA and PTMG are rather close to unity.

The glass transition temperature, $T_{\mathrm{g}}$, of PTMA and PTMG are calculated from VTF equation presented in Table II. We defined $T_{\mathrm{g}}$ as the temperature at which the segmental relaxation time equals $1 \mathrm{~s}$. The corresponding $T_{\mathrm{g}}$ were 171 and $165 \mathrm{~K}$ for PTMA and PTMG, respectively, close enough to that of semicrystalline PTMO $^{37}(178 \mathrm{~K})$. Cooperativity plots $\left(\tau_{2}\right.$ vs. $T_{\mathrm{g}} / T$ curves $\left.^{37}\right)$ were calculated for a liquid state of PTMA and PTMG. These plots in comparison with the cooperativity plot of semi-crystalline PTMO, taken from, ${ }^{37}$ are presented in Figure 10. Cooperativity plots obtained lie below plots of semi-crystalline PTMO and are steeper. 


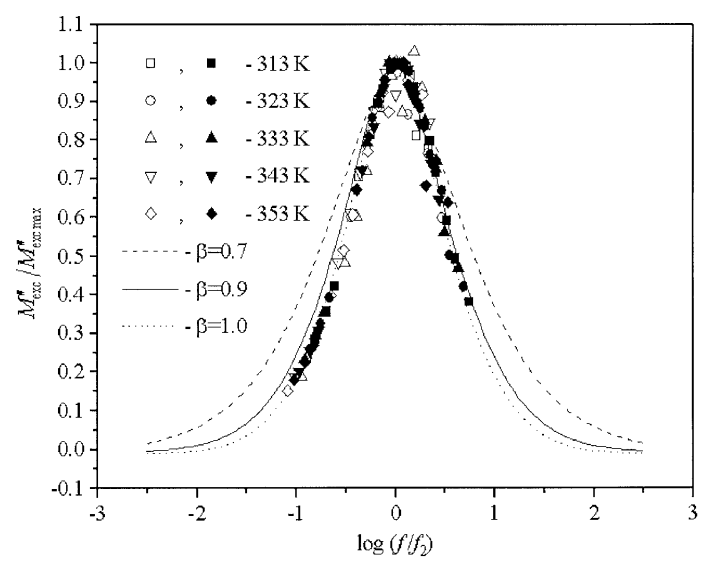

Figure 9. Normalised second maxima of the $M_{\mathrm{exc}}^{\prime \prime}$ spectra of the PTMA (open symbols) and PTMG (solid symbols) samples.

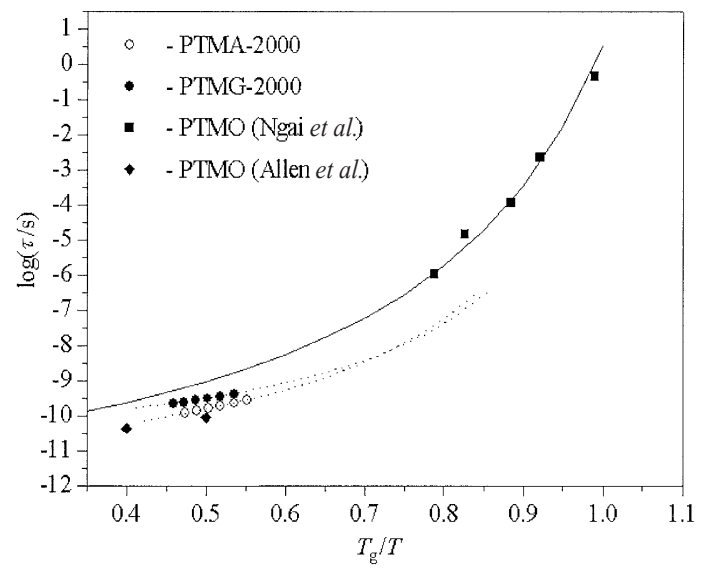

Figure 10. Segmental relaxation time as a function of normalized temperature, $T_{\mathrm{g}}$, for liquid PTMA $\left(T_{\mathrm{g}}=171 \mathrm{~K}\right)$ and PTMG $\left(T_{\mathrm{g}}=165 \mathrm{~K}\right)$ obtained by ultrasonic relaxation spectroscopy (this work) in comparison with the corresponding dependence for semicrystalline PTMO obtained by dielectric relaxation spectroscopy, 37 , and neutron spectroscopy, 35 .

\section{DISCUSSION}

\section{Types of Relaxation}

All the spectra shown in Figure 1 have similarities to those of the low molecular weight polyethers considered above. In Table I, characteristic frequencies of PTMG in the temperature region from $313 \mathrm{~K}$ to $333 \mathrm{~K}$ are $14-20 \mathrm{MHz}$ and $450-580 \mathrm{MHz}$ for the first and second dispersion region, respectively. It is interesting to compare the relaxation times observed with the corresponding data presented by the only paper ${ }^{5}$ on high frequency dielectrics of PPG of molecular weight 2000 at near-room temperatures.

If we compare PTMG relaxation times calculated using the characteristic frequency data presented in Table I with those presented by paper ${ }^{5}$ the low frequency region and at near-room temperatures the PPG data are about $10^{-8}$ and $10^{-10} \mathrm{~s}$ for low frequency and high fre- quency region, respectively. Closed relaxation times for both frequency regions are characteristic of PTMG and PTMA. Particular, what about the low frequency region, we have seen that the difference between them is not larger than $10-15 \%$. The corresponding difference for high frequency region is in the range 30 $50 \%$. Taking into account that PPG is partly similar but different from PTMG, dielectric and acoustic relaxation times of the first relaxation of PPG2000 and PTMG2000, respectively, are very close to say that the first relaxation process observed in the acoustic spectra of PTMG as well as PTMA are of RM type one. High frequency relaxation can be considered as the LSM, or structural relaxation, process.

Comparison of $\tau_{1}$ and $\tau_{2}$ against temperature and presented in Figures 4 showing of end group influence on the LSM region is more than on the RM relaxation region of the acoustic spectra of the PTMO-based low molecular weight polymers. The temperature behaviour of relaxation strength in the first relaxation region, $r_{1}$, in Figures 5a and 5b for PTMA and PTMG is the same and it is in line with that of most polymers. Consequently, whether or not molecular chain ends are H-bond connected, the RM relaxation related to equilibrium conformation transitions are practically the same. Relaxation times of structural relaxation as well as relaxation strength are thus more sensitive to the replacement of $\mathrm{OH}$ end groups with $\mathrm{CH}_{3}$ than those of large scale motion unaffected by such a replacement. This was completely confirmed by normal mode relaxation in $\mathrm{OH}$ group ended PPG as well as acylated one. ${ }^{6}$ The latter evidences that the effect of hydrogen bonding between the $\mathrm{OH}$ groups on the normal mode relaxation time is negligibly small.

A few intermolecular H-bonds radically slow down LSM relaxation (compare $f_{2}$ of PTMA and PTMG in Table I). In LSM relaxation region, decrease of relaxation strength is more pronounced for H-bond connected molecular chain (OH ended chain) in comparison with that of the free end one $\left(\mathrm{CH}_{3}\right.$ ended chain).

The steep rise of $M^{\prime}$ in PTMA and PTMG $M^{\prime} v s . f$ curves (Figure 6) that occurs only in the vicinity of $f_{2}$ at all the temperatures is certain to be of dynamic glass transition ( $\alpha$-relaxation) origin. This is good verification of the nature of the second relaxation process in the samples studied as of one related to the LSM mechanism.

\section{Thermoreological Simplicity/Complexity}

Temperature change leads to the change of the characteristic frequency in the wide frequency interval. If the time-temperature superposition can be applied to the processes under consideration, it should be possi- 
ble to convert the temperature domain experiment into the frequency domain one. The ultrasonic absorption data at any $T$ have to be superimposed exactly on the corresponding points taken at reference $T, T_{\text {ref }}$, by a horizontal displacement $\log \frac{f_{2}(T)}{f_{2}\left(T_{\text {ref }}\right)}$.

Sets of $M^{\prime \prime} v s . f$ isotherms in Figure 7 provide the possibility of analysis of first and second relaxations in the context of thermoreological simplicity/complexity alternative. ${ }^{37}$ In Figure 8, reduction to a reference temperature based on the position of the $M^{\prime \prime}$ second relaxation maximum leads to splitting of the curves reduced in the vicinity of the first relaxation maximum. The temperature behaviour of the $M^{\prime \prime} v s . f$ curves in the frequency range on first relaxation maximum and second one is different. Therefore, the thermoreological simplicity hypotheses is not applicable for the polymeric liquids studied.

For polyethers, only one absorption peak corresponding to dynamic glass transition (LSM, i.e., $\alpha$ relaxation), is usually observed in the temperature dependence of $M^{\prime \prime}$ at a constant frequency obtained by ultrasonical technique ${ }^{51,52}$ and no such absorption related to RM relaxation is present on the corresponding curves. This is in contrast with the present investigation on PTMO-based short chain polymers carried out showing two absorption maximuma at $5-2500 \mathrm{MHz}$. Obviously, ultrasonic temperature domain investigation of polyethers is useful to give a quick glimpse of all the temperature relaxation transition. However, the results obtained can lead to a loss of some peculiarities of the relaxation spectra and, what is more, cannot be used to a rigorous analysis of the data in the frame of the molecular dynamic models used.

\section{The Relaxation Spectra Parameters}

Most if not all dynamic processes in polymers should have a distribution of relaxation times. Not far above $T_{\mathrm{g}}$ and at long relaxation times a rather broad distribution of relaxation times was observed. ${ }^{53-55}$ Such "non-exponential decay" is explained by the coupling model. ${ }^{37}$

When the observed relaxations are due to single relaxation (Debye-like relaxations), the ultrasonic absorption coefficient can be represented by eqs 1 and 2 . This is the limit $\beta=1$ of eq 6 that describes the Debye case with a single relaxation time and after fitting the observed data by eq 2 we find parameters of a relaxation process in such an approximation.

From dielectric measurements, for polyethers such as PPG, the normal mode relaxation at near-room temperatures is practically Debye-like and the shape parameters for such processes were assumed to be near unity. ${ }^{5}$
In contrast to normal mode relaxation at the room temperature, LSM is usually characterised by wider distribution of relaxation time. For some liquids, such as ionic glass forming one, the broad distribution of relaxation times, as the material moves away from $T_{\mathrm{g}}$, sharpens up to become a single relaxation time in the high frequency region at elevated temperatures.$^{56}$ However, for short chain polyethers there is no general agreement regarding the temperature change of the relaxation time distribution. At room temperature Brillouin spectra of PPG, related to hindered LSM relaxation can be fitted to theoretical values using single relaxation time behaviour. ${ }^{57}$ The results of another Brillouin experiment as well as the near-room temperature dielectric measurements ${ }^{5}$ show that single relaxation time theory cannot explain LSM $\alpha$-relaxation behaviour of the polymer. ${ }^{10}$ For liquid PTMG, in accordance with Allen et al. using quasi-elastic neutron investigation, ${ }^{35} \beta$ was far smaller than unity at temperatures above the room temperature. Thus, for the polymers such as short chain polyethers there is no general agreement regarding the near-room temperature relaxation time distribution.

Modelling normalised $M_{\mathrm{exc}}^{\prime \prime} v s$. $f$ curves presented in Figure 9 show, at more than $120 \mathrm{~K}$ above the $T_{\mathrm{g}}$, for both PTMA and PTMG no broadening relaxation time of LSM relaxation. So in the near-room temperature range the LSM motions of these polyethers are characterised by a rather single relaxation time and the coupling parameter, $n=1-\beta$, tends to zero.

It was interesting to compare plots of the LSM relaxation of the liquid samples with the corresponding data obtained for liquid PTMO involved in the neutron spectroscopy investigation ${ }^{35}$ and cooperativity plot of semicrystalline PTMO ${ }^{37}$ Figure 10 shows that plots of PTMA and PTMG to lie just above the neutron spectroscopy data and well below plot of semi-crystalline PTMO.

\section{CONCLUSION}

Two relaxations were found from acoustical spectrum of the PTMO-based oligomer liquids with $\mathrm{CH}_{3}$ and $\mathrm{OH}$ end groups at $5-2500 \mathrm{MHz}$ and $313-353 \mathrm{~K}$. The higher relaxation times, $\tau_{1}$, as well as the lower relaxation times, $\tau_{2}$, are close to the relaxation time characteristic, at room temperature, of local segmental motion (so-called dynamic glass transition). The latter relaxation region precisely corresponds to only one marked sound velocity dispersion. Relaxation time and strength at different temperatures are sensitive to replacement of $\mathrm{OH}$ end groups with the $\mathrm{CH}_{3}$ while large scale motion is unaffected by such replacement. 
$\mathrm{OH}$ end groups and $\mathrm{H}$-bonding networks slow down local segmental motion relaxation and increase relaxation strength.

Using imaginary modulus against frequency curves, breakdown of thermoreological simplicity in the frequency range was found to occur and RM and LSM relaxations are Debye-like.

Temperatures studied the $\tau_{i} v$ s. inverse temperature dependency may be approximated by VTF equation. The corresponding $\tau_{1} v s .1 / T$ curves for $\mathrm{CH}_{3}$ and $\mathrm{OH}$ ended samples coincide but $\tau_{2}$ vs. $1 / T$ ones are very different. Plots drawn for the high frequency absorption region show that curves approximating $\tau_{2} v s . T_{\mathrm{g}} / T$ data are far below the corresponding curves obtained by K. L. Ngai and C. M. Roland for semi-crystalline PTMO and close enough to $\tau_{2} v s . T_{\mathrm{g}} / T$ data calculated using the neutron spectroscopy data obtained by Allen et al. for liquid PTMO.

Acknowledgment. This work was supported by INTAS 99-01852 project.

\section{REFERENCES}

1. M. E. Baur and W. H. Stockmayer, J. Chem. Phys., 43, 4319 (1965).

2. S. Yano, R. R. Rahalkar, S. P. Hunter, S. H. Wang, and R. H. Boyd, J. Polym. Sci., Polym. Phys. Ed., 14, 1877 (1976).

3. G. P. Johari, Polymer, 27, 866 (1986).

4. K. L. Ngai, A. Schonhals, and E. Schlosser, Macromolecules, 25, 4915 (1992).

5. K. Ichikawa, J. McKnight, R. Nozaki, T. K. Bose, and S. Yagihara, Polymer, 35, 1166 (1994).

6. T. Hayakawa and K. Adachi, Polymer, 42, 1725 (2001).

7. C. H. Wang and Y. Y. Huang, Macromolecules, 14, 1363 (1981)

8. H. H. Krbecek, W. Kupish, and M. Pietrralla, Polymer, 37, 3483 (1996).

9. B. D. Freeman, L. Bokobza, P. Sergot, and L. Monneri, Macromolecules, 23, 2566 (1990).

10. L. Borjesson, J. R. Stevens, and L. M. Torell, Polymer, 28, 1803 (1987).

11. I. Alig, E. Donth, W. Schenk, S. Hoering, and Ch. Wohlfart, Polymer, 29, 2081 (1988).

12. G. Fleischer, M. Helmstedt, and I. Alig, Polym. Commun., 31, 409 (1990).

13. R. D. de la Batie, F. Laupretre, and L. Monnerie, Macromolecules, 21, 2052 (1988)

14. G. Allen, J. S. Higgins, and C. J. Wright, J. Chem. Soc., Faraday Trans., 2, 70, 348 (1974).

15. G. Allen, J. S. Higgins, and A. Maconnachie, J. Chem. Soc., Faraday Trans., 2, 78, 2117 (1982).

16. S. B. Grigor'ev, S. A. Kukorina, Ju. S. Manucarov, I. G. Michailov, Vestnik Leningr. Univ. (Bulletin of Leningrad University), 4, 112 (1981).

17. I. Alig, S. B. Grigor'ev, Ju. S. Manucarov, and S. A. Manu- carva, Acta Polymerica, 37, 698 (1986).

18. I. Alig, S. B. Grigor'ev, Ju. S. Manucarov, and S. A. Manucarova, Acta Polymerica, 37, 733 (1986).

19. A. A. Chuiko, V. S. Sperkach, V. M. Ogenko, T. P. Tantsyura, L. N. Ganyuk, L. V. Dubrovina, and A. L. Stribulevich, Polymer Science, Series A, 33, 1155 (1991).

20. A. A. Chuiko, V. S. Sperkach, V. M. Ogenko, T. P. Tantsyura, L. N. Ganyuk, L. V. Dubrovina, and B. T. Yeleusinov, Polymer Science, Series A, 33, 1160 (1991).

21. M. Xu, E. M. Eyring, and S. Petrucci, J. Chem. Soc., Faraday Trans., 2, 92, 4969 (1996).

22. A. J. Barlow and A. Erginsav, Polymer, 16, 110 (1975).

23. T. Alper, A. J. Barlow, and R. W. Gray, Polymer, 17, 665 (1976).

24. J. Cocrane, G. Harrison, J. Lamb, and D. W. Phillips, Polymer, 37, 837 (1980).

25. N. Koizumi, J. Chem. Phys., 27, 625 (1957).

26. E. Wessling, M. Stockhausen, and G. Schulz, J. Mol. Liq., 49, 105 (1991).

27. M. Stockhausen, M. Abd-El-Rehim, Z. Naturforsch. A: Phys. Sci., 49, 1229 (1994).

28. G. Allen, T. M. Connor, and H. Pursey, Trans. Faraday Soc., 59,1525 (1963).

29. L. M. Torell, P. Jacobson, D. Sidebottom, and G. Petersen, Solid State Ionics, 53, 1037 (1992).

30. S. B. Grigoryev, Yu. S. Manucharov, and S. A. Manucharova, Polymer Science, Series B, 18, 150 (1986).

31. A. L. Striboulevich, V. S. Sperkach, Ya. V. Sperkach, Polym. Eng. Sci., 39, 394 (1999).

32. M. Salomon, M. Xu, E. Eyring, and S. Petrucchi, J. Phys. Chem., 98, 8234 (1994).

33. G. Allen, J. S. Higgins, A. Maconnachie, and R. E. Ghosh, J. Chem. Soc., Faraday Trans. 2, 78, 2117 (1982).

34. R. E. Wetton and G. Williams, Trans. Faraday Soc., 61, 2132 (1965).

35. K. L. Ngai and C. M. Roland, Macromolecules, 26, 6824 (1993).

36. R. E. Wetton and G. Allen, Polymer, 17, 331 (1966).

37. K. L. Ngai and D. J. Plazek, Rubber Chem. Technol., 68, 376 (1995).

38. W. H. Stockmayer, Pure Appl. Chem., 15, 539 (1967).

39. Yu. S. Lipatov, Yu. Yu. Kercha, L. M. Sergeeva, "Polyuretrhane Structure and Properties", Naukova Dumka, Kiev, 1970 (in Russian).

40. D. Dieterich, W. Keberle, and H. Witt, Angew. Chem., 82, 53 (1970).

41. D. Feng, G. L. Wilkes, B. Lee, and J. E. McGrath, Polymer, 33, 526 (1992).

42. V. V. Shilov, V. V. Shevchenko, P. Pissis, A. Kyritsis, G. Georgoussis, Yu. P. Gomza, S. D. Nesin, and N. S. Klimenko, J. Non-Cryst. Solids, 275, 116 (2000).

43. X. Wang, H. Li, X. Tang, and F.-C. Chang, J. Polym. Sci., Part B: Polym. Phys., 37, 837 (1999).

44. G. Heinrich, I. Alig, and E. Donth, Polymer, 29, 1198 (1988).

45. V. S. Sperkach, "The Molecular Mechanisms of Acoustic Relaxation in Liquids", D. Sci. Thesis in Physics and Mathematics, Tashkent University, Tashkent, 1991.

46. H. Farber and S. Petrucci, in 'Ultrasonic Absorption Spectrometry', "The Chemical Physics of Solution”, R. R. Dog- 
onadze, Ed., Elsevier Science Publisher B.V., Amsterdam, 1986, Part B, chapt. 9.

47. J. Eschmann, J. Strasser, M. Xu, Y. Okamoto, E. M. Eyring, and S. Petrucci, J. Phys. Chem., 94, 3908 (1990).

48. A. Rupprecht and U. Kaatze, J. Phys. Chem. A, 101, 9884 (1997).

49. T. A. Litovitz, and C. Davis, in "Physical Acoustics. Principle and Methods", W. P. Mason, Ed., vol. II, part A, Properies of Gases, Liquids and Solutions, Academic Press Inc., New York and London, 1965, chapt. 5.

50. G. Williams and D. C. Watts, Trans. Faraday Soc., 66, 80 (1970).
51. P.-Y. Baillif, M. Tabellout, and J. Emery, Macromolecules, 32, 3432 (1999).

52. I. Alig, F. Stieber, and S. Wartewig, Polymer, 32, 2146 (1991).

53. K. L. Ngai, D. J. Plazek, and S. S. Deo, Macromolecules, 20, 3047 (1987).

54. J. Colmenero, A. Alegria, and J. M. Alberdi, J. Non-Cryst. Solids, 131-133, 949 (1991).

55. J. C. Phillips, Rep. Prog. Phys., 28, 1133 (1996).

56. K. L. Ngai and H. Jain, Solid State Ionics, 18-19, 362 (1986).

57. R. Bergman, A. Brodin, D. Engberg, Q. Lu, C. A. Angell, and L. M. Torell, Electrochimica Acta, 13-14, 2049 (1995). 